\title{
Determining the focal mechanisms of the events in the Carpathian region of Ukraine
}

\author{
A. Pavlova, O. Hrytsai, and D. Malytskyy \\ Carpathian Branch of the Institute of Geophysics named after S. I. Subbotin NAS of Ukraine, Lviv, Ukraine \\ Correspondence to: A. Pavlova (susyinet@gmail.com)
}

Received: 24 December 2013 - Published in Geosci. Instrum. Method. Data Syst. Discuss.: 5 March 2014

Revised: 15 October 2014 - Accepted: 16 October 2014 - Published: 30 October 2014

\begin{abstract}
The modification of the matrix method for constructing the displacement field on the free surface of an anisotropic layered medium is presented. The source of seismic waves is modelled by a randomly oriented force and seismic tensor. A trial and error method is presented for solving the inverse problem of determining parameters of the earthquake source. A number of analytical and numerical approaches to determining the earthquake source parameters, based on the direct problem solutions, are proposed. The focal mechanisms for the events in the Carpathian region of Ukraine are determined by the graphical method. The theory of determination of the angles of orientation of the fault plane and the earthquake's focal mechanism are presented. The focal mechanisms obtained by two different methods are compared.
\end{abstract}

\section{Introduction}

The main data sources in seismology are the seismic records of natural or man-made events that are received on the earth's surface. The task of modern seismic analysis is to obtain the maximum possible information about the nature of wavefield propagation. Solving these problems involves the study of seismic regions of Ukraine and interpretation of wave fields in order to determine the earthquake focal mechanisms.

In recent years one of the most important methods has been the development of approaches for constructing the theoretical seismograms, which allow the study of the structure of the medium and determination of the earthquake source parameters. The effects on the wave field and seismic wave propagation in the earth's interior should be considered when calculating these seismograms. Thus, the displacement field, which is registered on the free surface of an inhomogeneous medium, depends on the model of the geological structure and the physical processes in the source.

Interpretation of seismic research can predict the dynamic properties of elastic media, and consider the effects of anisotropy in the inversion problems of determining the source parameters. Therefore, the problem of mathematical modelling of seismic wave propagation in an anisotropic medium is relevant. Over the past decade considerable experience in theoretical and algorithmic solutions of a wide range of dynamic seismology problems has accumulated. There are plenty of methods for solving such problems, which are quite effectively used in geophysics, including seismology. Analytical problem solving methods are developed only for a relatively narrow range of tasks. More precise and hence more complex mathematical models are implemented by numerical methods. The latter give a solution only in certain limited areas of model medium, and this is the main drawback of numerical methods. This means that the use of numerical methods, including finite difference method (Fuchs, 1977; Ilan, 1975; Bullen, 1953; Yang et al., 2002; Zahradnik, 1975) and finite element method (Thomson, 1950; Woodhouse, 1978) for modelling of seismic wave propagation in inhomogeneous anisotropic media, gives very high accuracy results but requires a grid which covers the entire area occupied by the investigated object and a significant number of computer resources for the solution of high-dimensional systems of algebraic equations. Therefore, it is difficult to implement, even with the use of modern computational tools, including clusters. The matrix method is used to obtain solutions which avoid the complicated procedures to satisfy all boundary conditions. The usefulness of solutions obtained by this 


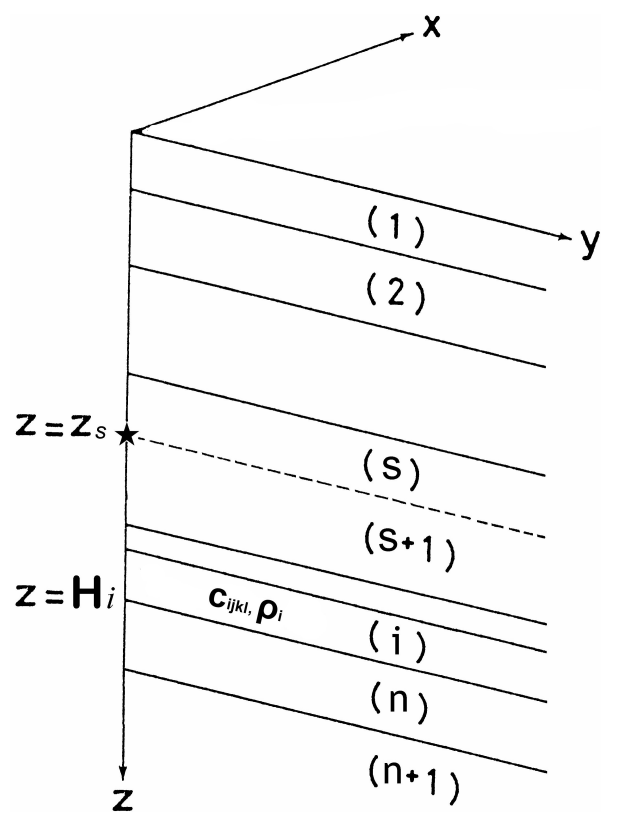

Figure 1. Model vertically inhomogeneous medium.

method is considered in Babuska (1981), Bachman (1979), Backus (1962), Behrens (1967), and Dunkin (1965). The matrix method allows for a common approach to examine the propagation of waves in a wide class of systems. This method allows obtaining solutions in a more compact and convenient form for further analytical and numerical calculations.

In the 1950s, Thomson (1950) and Haskell (1953) first proposed a method for constructing interference fields by simulation of elastic waves in layered isotropic half-space with planar boundaries (Haskell, 1953). The matrix method was developed in a number of previous studies (Malytskyy, 1998, 2010; Malytskyy and Muyla, 2008; Kennett, 1972, 1983; Cerveny, 2001; Chapman, 2004). The stable algorithms of seismogram calculation for all angles of seismic wave propagation are obtained. The matrix method is generalized for low-frequency waves in inhomogeneous, elastic, concentric cylindrical and spherical layers surrounded by an elastic medium. The concept of the characteristic matrix determined by physical parameters of the environment is developed. The matrix method is used for seismic wave propagation in elastic, liquid and thermoelastic media. In addition, it has been generalized for the study of other processes described by linear equations. The advantage of the matrix method is the ability to compactly write matrix expressions that are useful both in analytical studies and numerical calculations.

The matrix method and its modifications are used to simulate the seismic wave propagation in isotropic and anisotropic media. This method is quite comfortable and has several advantages over other approaches. Both advantages and disadvantages of the matrix method are well described

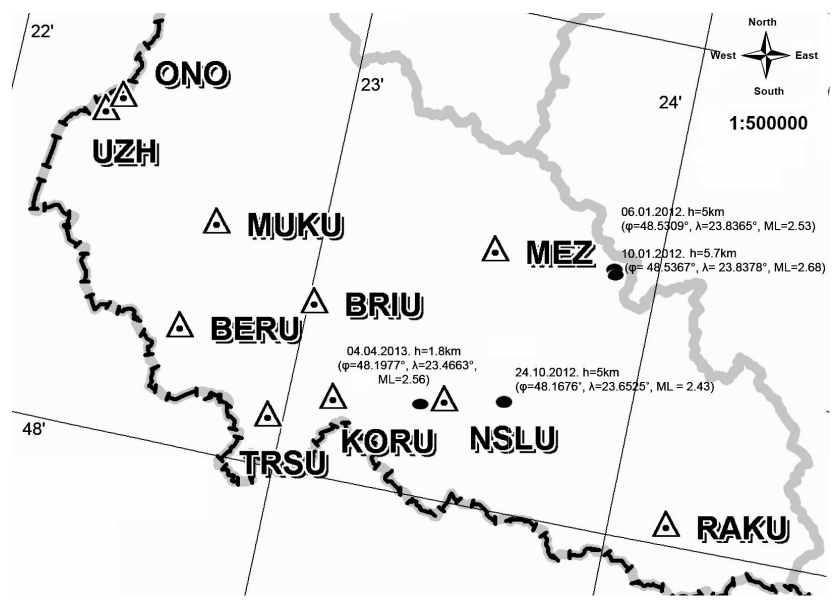

Figure 2. Location map of the projection of seismic stations in the Carpathian region of Ukraine and specified epicentre of the four events in the Carpathian region of Ukraine.

in Malytskyy (2010), Thomson (1950), Ursin (1983), and Thomsen (1986).

Today in seismology much attention is given to mathematical modelling as one of the main tools for the analysis and interpretation of the wave fields. In this paper using a modification of the Thomson-Haskell matrix method, rigorous equations for the wave field on the free surface of inhomogeneous anisotropic medium are obtained, when a source of seismic waves is located within a homogeneous anisotropic layer and presented by the seismic moment tensor. Note that the problem of wave field modelling generated by a source, which is presented in terms of seismic moment tensor, also has practical applications in seismology. Using this method, the approaches to determining the displacement field are developed for different types of earthquake sources, which will be shown in the following sections.

\section{Direct problem}

The problem of wave field modelling, when the source is presented by seismic tensor moment, has practical applications in seismology. Therefore, the development of methods for determining the displacement field on the free surface of an anisotropic inhomogeneous medium for sources of this type is an actual task and needs to be resolved.

In this section the propagation of seismic waves in an inhomogeneous anisotropic medium is considered. The modification of the matrix method of construction of wave field on the free surface of an anisotropic medium is presented. The earthquake source represented by a randomly oriented force or a seismic moment tensor is placed on an arbitrary boundary of a layered anisotropic medium. The theory of the matrix propagator in a homogeneous anisotropic medium by introducing a "wave propagator" is presented. It is shown 


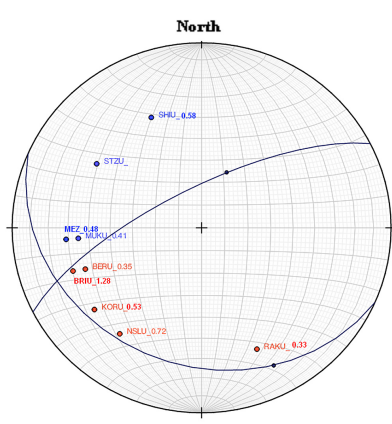

a

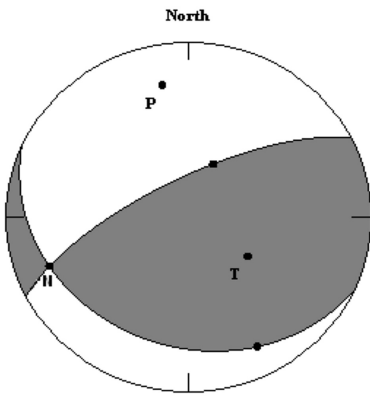

b

Figure 3. the most probable focal mechanism of event 6 January 2012: (a) location of the projection of seismic stations and nodal planes according to the input data (Table 3) and (b) focal mechanism of event 6 January 2012 determined by the graphic method.

that for an anisotropic layered medium the matrix propagator can be represented by a "wave propagator" in each layer. The displacement field on the free surface of an anisotropic medium is obtained from the received system of equations considering the radiation condition and that the free surface is stressless.

\subsection{Theory of modification of the matrix method}

The problem of wave field modelling, when the source is presented by seismic moment, has practical applications in seismology. Therefore, the development of methods for determining the displacement field on the free surface of an anisotropic inhomogeneous medium for sources of this type is an actual task and needs to be resolved.

In this paper the propagation of seismic waves in an anisotropic inhomogeneous medium is modelled by a system of homogeneous anisotropic layers, as shown in Fig. 1. Each layer is characterized by the propagation velocity of Pand S-wave and density. At the boundaries between layers hard contact condition is met, except for the border, where the source of seismic waves is located.

The earthquake source is modelled by nine pairs of forces, which represent a seismic moment tensor. This description of the point source is sufficiently known and effective for simulation of seismic waves in layered half-space (Haskell, 1953). In general, the source is also assumed to be distributed over time: seismic moment $M_{0}(\mathrm{t})$ is a function of time. This means that the physical process in the source does not occur instantaneously - but within a certain time frame. It is known for our seismic events $\left(M_{\mathrm{W}} \sim 2-3\right)$ that the time during which the event occurred may be $0.1-0.7 \mathrm{~s}$. The determination of the source time function is an important seismic problem. In this chapter the direct problem solution is shown, when a point source is located on an arbitrary boundary of layered anisotropic media.

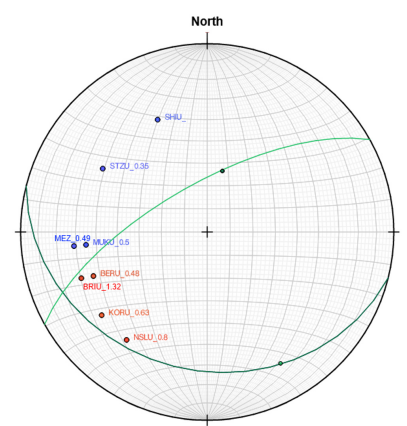

a

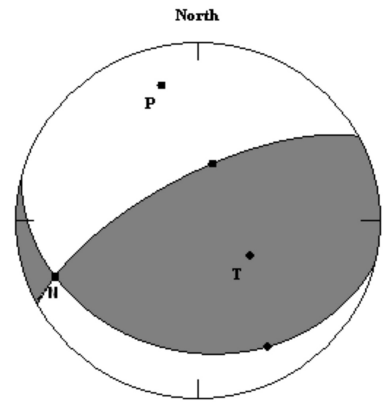

b
Figure 4. The most probable focal mechanism of event 10 January 2012: (a) Location of the projection of seismic stations and nodal planes according to the input data (Table 4), and (b) focal mechanism of event 10 January 2012 determined by the graphic method.

Table 1. Velocity model for the Carpathian region of Ukraine.

\begin{tabular}{lrrr}
\hline $\begin{array}{l}\text { Depth, } \\
\mathrm{km}\end{array}$ & $\begin{array}{r}\text { Velocity } \\
\text { of P-wave, } \\
\mathrm{km} \mathrm{s}^{-1}\end{array}$ & $\begin{array}{r}\text { Velocity } \\
\text { of S-wave, } \\
\mathrm{km} \mathrm{s}^{-1}\end{array}$ & $\begin{array}{r}\text { Density, } \\
\mathrm{kg} \mathrm{m}^{-3}\end{array}$ \\
\hline 0 & 4.70 & 2.71 & 2200 \\
2.5 & 5.50 & 3.17 & 2350 \\
6.5 & 6.30 & 3.64 & 2500 \\
8.0 & 6.10 & 3.52 & 2570 \\
12.0 & 6.70 & 3.87 & 2640 \\
17.5 & 6.85 & 3.95 & 2690 \\
21.0 & 6.40 & 3.70 & 2720 \\
26.5 & 8.10 & 4.68 & 2800 \\
\hline
\end{tabular}

We assume the usual linear relationship between stress $\tau_{i j}$ and strain $\mathbf{e}_{k l}$

$\tau_{i j}=\mathbf{c}_{i j k l} \mathbf{e}_{k l}=\mathbf{c}_{i j k l} \frac{\partial u_{k}}{\partial x_{l}}$,

where $\boldsymbol{u}=\left(u_{x}, u_{y}, u_{z}\right)^{T}$ is the displacement vector.

The equation of motion for an elastic homogeneous anisotropic medium, in the absence of body forces, is that of Fryer and Frazer (1984)

$\rho \frac{\partial^{2} u_{i}}{\partial t^{2}}=\mathbf{c}_{i j k l} \frac{\partial^{2} u_{l}}{\partial x_{i} \partial x_{k}}$,

where $\rho$ is the uniform mass density, and $\mathbf{c}_{i j k l}$ are the elements of the uniform elastic coefficient tensor.

Taking the Fourier transform of Eqs. (1) and (2), we obtain the matrix equation (Fryer and Frazer, 1987):

$\frac{\partial b}{\partial z}=j \omega \mathbf{A}(z) b(z)$,

where $\boldsymbol{b}=(u, \tau)^{T}$ is the vector of displacement and scaled traction $\tau=-\frac{1}{j \omega}\left(\tau_{x z}, \tau_{y z}, \tau_{z z}\right)^{T}$. With the definition of $\boldsymbol{b}$, the 
Table 2. Spectral parameters for the events in the Carpathian region of Ukraine calculated by Eqs. (12)-(19).

\begin{tabular}{lrrrrrrrr}
\hline Event & $M_{0}, \mathrm{Nm}$ & $f_{c p}, \mathrm{~Hz}$ & $R, \mathrm{~m}$ & $A, \mathrm{~m}^{2}, 10^{5}$ & $\bar{D}, \mathrm{~mm}$ & $\Delta \sigma, \mathrm{MPa}$ & $E_{\mathrm{s}}, \mathrm{MJ}$ & $\mathrm{ML}$ \\
\hline 6 Jan 2012 & $2.22 \times 10^{13}$ & 7.67 & 220 & 1.54 & 6.2 & 0.895 & 355 & 2.53 \\
10 Jan 2012 & $4.24 \times 10^{13}$ & 7.38 & 230.09 & 1.66 & 10.9 & 1.52 & 678 & 2.68 \\
24 Oct 2012 & $1.72 \times 10^{13}$ & 7.87 & 215.77 & 1.46 & 4.4 & 0.065 & 240 & 2.43 \\
4 Apr 2013 & $2.55 \times 10^{13}$ & 6.87 & 211.22 & 1.4 & 11 & 1.18 & 408 & 2.56 \\
\hline
\end{tabular}

Table 3. Input data for determining the focal mechanism of event 6 January 2012.

\begin{tabular}{lrrrr}
\hline Stations & $\begin{array}{r}\text { Sign of } \\
\text { first arrival }\end{array}$ & Azimuth, ${ }^{\circ}$ & $\begin{array}{r}\text { Take-off } \\
\text { angle, }\end{array}$ & $\begin{array}{r}\text { lg } \\
\text { As/Ap }\end{array}$ \\
\hline MEZ & - & 265.1 & 29 & 0.48 \\
NSLU & + & 217.2 & 29 & 0.72 \\
RAKU & + & 156.4 & 29 & 0.33 \\
BRIU & + & 250.7 & 29 & 1.28 \\
KORU & + & 231.6 & 29 & 0.53 \\
SHIU & - & 335.8 & 35 & 0.58 \\
MUKU & - & 264.7 & 35 & 0.41 \\
BERU & + & 249.9 & 35 & 0.35 \\
STZU & - & 301.7 & 35 & \\
\hline
\end{tabular}

Table 4. Input data for the determining the focal mechanism of event 10 January 2012.

\begin{tabular}{lrrrr}
\hline Stations & $\begin{array}{r}\text { Sign of } \\
\text { first arrival }\end{array}$ & Azimuth, ${ }^{\circ}$ & $\begin{array}{r}\text { Take-off } \\
\text { angle, }\end{array}$ & $\begin{array}{r}\text { lg } \\
\text { As/Ap }\end{array}$ \\
\hline MEZ & - & 263.6 & 29 & 0.49 \\
NSLU & + & 216.9 & 29 & 0.8 \\
BRIU & + & 250.2 & 29 & 1.32 \\
KORU & + & 231.2 & 29 & 0.63 \\
SHIU & - & 335.6 & 35 & - \\
MUKU & - & 264.2 & 35 & 0.5 \\
BERU & + & 249.5 & 35 & 0.48 \\
STZU & - & 301.4 & 35 & 0.35 \\
\hline
\end{tabular}

system matrix $\mathbf{A}$ has the structure $\mathbf{A}=\left(\begin{array}{cc}\mathbf{T} & \mathbf{C} \\ \mathbf{S} & \mathbf{T}^{T}\end{array}\right)$, where $\mathbf{T}, \mathbf{S}$ and $\mathbf{C}$ are $3 \times 3$ submatrices, and $\mathbf{C}$ and $\mathbf{S}$ are symmetric.

For any vertically stratified medium, the differential system (Eq. 3) can be solved subject to specified boundary conditions to obtain the response vector $\boldsymbol{b}$ at any desired depth. If the response at depth $z_{0}$ is $\boldsymbol{b}\left(z_{0}\right)$, the response at depth $z$ is

$\boldsymbol{b}(z)=\mathbf{P}\left(z, z_{0}\right) b\left(z_{0}\right)$

where $\mathbf{P}\left(z, z_{0}\right)$ is the stress-displacement propagator.

To find this propagator, it is necessary to find the eigenvalues (vertical slownesses), the eigenvector matrix $\mathbf{D}$, and its inverse $\mathbf{D}^{-1}$ (Fryer and Frazer, 1984):

$b(z)=\mathbf{D Q}\left(z, z_{1}\right) \mathbf{D}^{-1} b\left(z_{1}\right)$,
Table 5. Input data for the determining the focal mechanism of event 24 October 2012.

\begin{tabular}{lrrrr}
\hline Stations & $\begin{array}{r}\text { Sign of } \\
\text { first arrival }\end{array}$ & Azimuth, ${ }^{\circ}$ & $\begin{array}{r}\text { Take-off } \\
\text { angle, }\end{array}$ & $\begin{array}{r}l g \\
\text { As / Ap }\end{array}$ \\
\hline NSLU & - & 283 & -11 & 0.29 \\
KORU & + & 268 & 29 & 1.06 \\
MEZ & - & 345 & 29 & 0.44 \\
RAKU & - & 112 & 29 & 0.56 \\
BRIU & + & 292 & 29 & 0.69 \\
TRSU & + & 261 & 29 & 0.82 \\
BERU & + & 276 & 29 & 0.75 \\
MUKU & + & 294 & 29 & 0.72 \\
\hline
\end{tabular}

Table 6. Input data for the determining the focal mechanism of event 4 April 2013. "e" indicates "inaccurate" arrival of P-wave.

\begin{tabular}{lrrrr}
\hline Stations & $\begin{array}{r}\text { Sign of } \\
\text { first arrival }\end{array}$ & Azimuth, ${ }^{\circ}$ & $\begin{array}{r}\text { Take-off } \\
\text { angle, }\end{array}$ & $\begin{array}{r}l g \\
\text { As/Ap }\end{array}$ \\
\hline NSLU & + & 269 & -53 & - \\
KORU & - & 260 & 31 & 0.43 \\
MEZ & - & 6 & 31 & 0.084 \\
BRIU & - & 295 & 42 & 0.65 \\
TRSU & - & 253 & 42 & 0.57 \\
BERU & $\mathrm{e}$ & 274 & 42 & 2.64 \\
MUKU & - & 297 & 42 & 0.71 \\
UZH & - & 299 & 45 & 0.88 \\
\hline
\end{tabular}

where $\mathbf{Q}$ is the "wave" propagator (Fryer and Frazer, 1984):

$\mathbf{Q}\left(z, z_{1}\right)=\left(\begin{array}{cc}\mathbf{E}_{u} & 0 \\ 0 & \mathbf{E}_{D}\end{array}\right)$,

where $\mathbf{E}_{u}=\operatorname{diag}\left[e^{j \omega\left(z-z_{1}\right) q_{p}^{u}}, e^{j \omega\left(z-z_{1}\right) q_{s_{1}}^{u}}, e^{j \omega\left(z-z_{1}\right) q_{s_{2}}^{u}}\right]$, and $\mathbf{E}_{D}=\operatorname{diag}\left[e^{j \omega\left(z-z_{1}\right) q_{p}^{D}}, e^{j \omega\left(z-z_{1}\right) q_{s_{1}}^{D}}, e^{j \omega\left(z-z_{1}\right) q_{s_{2}}^{D}}\right]$.

In the isotropic case the eigenvector matrix $\mathbf{D}$ is known analytically, so the construction of the propagator is straightforward. In the anisotropic case, analytic solutions have been found only for simple symmetries, so in general solutions will be found numerically.

The layered anisotropic medium, which consists of $n$ homogeneous anisotropic layers on an anisotropic half-space $(n+1)$ (Fig. 1), is considered. The source in the form of a jump in the displacement-stress $b_{s+1}-b_{s}=F$ is placed on 
Table 7. Parameters of the focal mechanisms determined by the graphic method.

\begin{tabular}{|c|c|c|c|c|c|c|c|c|c|c|c|c|}
\hline \multirow{2}{*}{ Event } & \multicolumn{3}{|c|}{ Plane 1} & \multicolumn{3}{|c|}{ Plane 2} & \multicolumn{2}{|c|}{$P$} & \multicolumn{2}{|c|}{$T$} & \multicolumn{2}{|c|}{$N$} \\
\hline & $\varphi_{\mathrm{s}}$ & $\delta$ & $\lambda$ & $\varphi_{\mathrm{s}}$ & $\delta$ & $\lambda$ & Azm & Plunge & Azm & Plunge & Azm & Plunge \\
\hline 6 Jan 2012 & $243^{\circ}$ & $72^{\circ}$ & $69^{\circ}$ & $114^{\circ}$ & $27^{\circ}$ & $138^{\circ}$ & $349^{\circ}$ & $24^{\circ}$ & $125^{\circ}$ & $58^{\circ}$ & $250^{\circ}$ & $20^{\circ}$ \\
\hline 10 Jan 2012 & $104^{\circ}$ & $27^{\circ}$ & $129^{\circ}$ & $241^{\circ}$ & $69^{\circ}$ & $72^{\circ}$ & $345^{\circ}$ & $22^{\circ}$ & $125^{\circ}$ & $62^{\circ}$ & $248^{\circ}$ & $17^{\circ}$ \\
\hline 20 Oct 2012 & $170^{\circ}$ & $27^{\circ}$ & $131^{\circ}$ & $316^{\circ}$ & $67^{\circ}$ & $75^{\circ}$ & $201^{\circ}$ & $65^{\circ}$ & $57^{\circ}$ & $21^{\circ}$ & $322^{\circ}$ & $13^{\circ}$ \\
\hline 4 Apr 2013 & $174^{\circ}$ & $45^{\circ}$ & $173^{\circ}$ & $269^{\circ}$ & $85^{\circ}$ & $45^{\circ}$ & $33^{\circ}$ & $27^{\circ}$ & $142^{\circ}$ & $34^{\circ}$ & $274^{\circ}$ & $44^{\circ}$ \\
\hline
\end{tabular}

Table 8. Parameters of the average variants of the focal mechanisms determined by the trial and error method.

\begin{tabular}{|c|c|c|c|c|c|c|c|c|c|c|}
\hline \multirow{2}{*}{ Event } & \multicolumn{3}{|c|}{ Plane 1} & \multicolumn{3}{|c|}{ Plane 2} & \multicolumn{2}{|c|}{$P$} & \multicolumn{2}{|c|}{$T$} \\
\hline & $\varphi_{\mathrm{s}}$ & $\delta$ & $\lambda$ & $\varphi_{\mathrm{S}}$ & $\delta$ & $\lambda$ & Azm & Plunge & Azm & Plunge \\
\hline 6 Jan 2012 & 89 & 10 & 102 & 257 & 80 & 88 & 169 & 35 & 344 & 55 \\
\hline 10 Jan 2012 & 90 & 10 & 102 & 258 & 80 & 88 & 169 & 35 & 345 & 55 \\
\hline 24 Oct 2012 & 152 & 20 & 112 & 309 & 71 & 82 & 225 & 26 & 27 & 63 \\
\hline 4 Apr 2013 & 173 & 43 & 173 & 268 & 85 & 48 & 210 & 28 & 323 & 36 \\
\hline
\end{tabular}

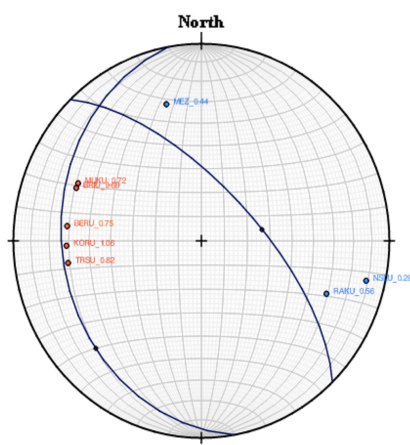

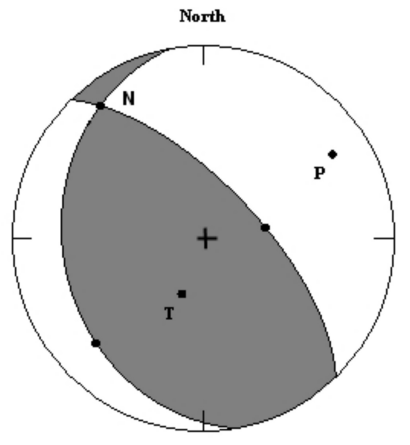

Figure 5. The most probable focal mechanism of event 24 October 2012: (a) location of the projection of seismic stations and nodal planes according to the input data (Table 5) and (b) focal mechanism of event 24 October 2012 determined by the graphic method.

the s-boundary (Fig. 1); it is easy to write the following matrix equation, using Eq. (5)-(6):

$b_{n+1}=\left.\mathbf{P}_{n, s} b_{s+1}\right|_{z=z_{s}}, v_{n+1}=\mathbf{D}_{n+1}^{-1} \mathbf{D}_{n} \mathbf{Q}_{n} \mathbf{D}_{n}^{-1} \ldots \mathbf{D}_{s+1}$

$\left.\mathbf{Q}_{s+1} \mathbf{D}_{s+1}^{-1} \cdot b_{s+1}\right|_{z=z_{s}}$,

$\left.b_{s}\right|_{z=z_{s}}=\mathbf{P}_{s, s-1} \mathbf{P}_{s-1, s-2 \ldots} \ldots \mathbf{P}_{2,1} \mathbf{P}_{1,0} \cdot b_{0}=\mathbf{D}_{s} \mathbf{Q}_{s} \mathbf{D}_{s}^{-1} \ldots \mathbf{D}_{1}$

$\mathbf{Q}_{1} \mathbf{D}_{1}^{-1} \cdot b_{0}$,

$v_{n+1}=\mathbf{D}_{n} \mathbf{Q}_{n} \mathbf{D}_{n}^{-1} \ldots \mathbf{D}_{s+1} \mathbf{Q}_{s+1} \mathbf{D}_{s+1}^{-1} \cdot\left(b_{s}+F\right)$

$=\mathbf{G}^{n}+1, \mathbf{s}+1 \cdot\left(\mathbf{G}_{s, 1} b_{0}+F\right)=$

$\mathbf{G}^{n}+1, s+1 \mathbf{G}_{s, 1} b_{0}+\mathbf{G}^{n+1, s+1} \cdot F=\mathbf{G} b_{0}+\mathbf{G}^{n}+1, s+1 \cdot F$

$v_{n+1}=\mathbf{G} b_{0}+\mathbf{G} \cdot \mathbf{G}_{s, 1}^{-1} \cdot F=\mathbf{G}\left(b_{0}+\mathbf{G}_{s, 1}^{-1} \cdot F\right)=\mathbf{G}\left(b_{0}+\tilde{F}\right)$,

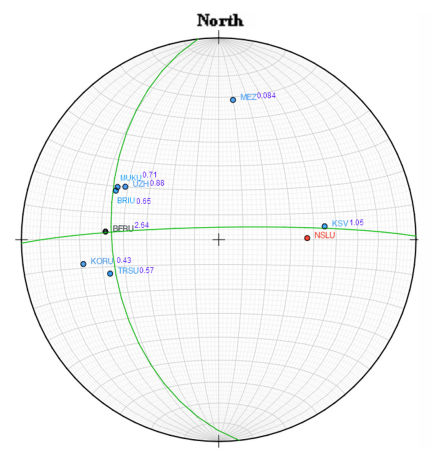

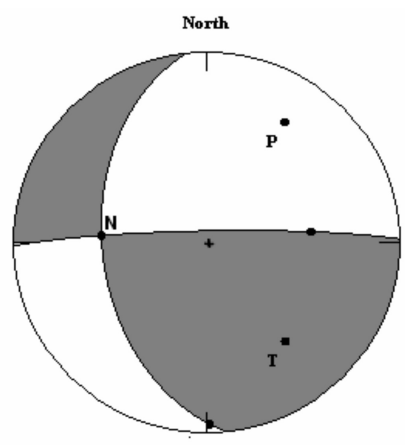

Figure 6. The most probable focal mechanism of event 4 April 2013: (a) location of the projection of seismic stations and nodal planes according to the input data (Table 6), and (b) focal mechanism of event 4 April 2013 determined by the graphic method.

where $\mathbf{G}=\mathbf{D}_{n+1}^{-1} \mathbf{D}_{n} \mathbf{Q}_{n} \mathbf{D}_{n}^{-1} \ldots \mathbf{D}_{s+1} \mathbf{Q}_{s+1} \mathbf{D}_{s+1}^{-1} \ldots \mathbf{D}_{2}^{-1} \mathbf{D}_{1} \mathbf{Q}_{1} \mathbf{D}_{1}^{-1}$ is the characteristic matrix of a layered anisotropic medium.

$v_{n+1}=\mathbf{D}_{n}+1^{-1} \mathbf{D}_{n} \mathbf{Q}_{n} \mathbf{D}_{n}^{-1} \cdots \mathbf{D}_{2}^{-1} \mathbf{D}_{1} \mathbf{Q}_{1} \mathbf{D}_{1}^{-1} b_{0}$,

where $\tilde{F}=\mathbf{G}_{s, 1}^{-1} \cdot F, \mathbf{G}=\mathbf{G}^{n+1, s+1} \cdot \mathbf{G}_{s, 1}$.

Using Eq. (7) and the radiation condition (with a halfspace $(n+1)$ the waves are not returned), and also the fact that the tension on the free surface equals to zero, we obtain a system of equations:

$\left(\begin{array}{l}0 \\ 0 \\ 0 \\ v_{D}^{P} \\ D_{1} \\ v_{D} \\ J_{2} \\ v_{D}\end{array}\right)=\left(\begin{array}{llllll}G_{11} & G_{12} & G_{13} & G_{14} & G_{15} & G_{16} \\ G_{21} & G_{22} & G_{23} & G_{24} & G_{25} & G_{26} \\ G_{31} & G_{32} & G_{33} & G_{34} & G_{33} & G_{36} \\ G_{41} & G_{42} & G_{43} & G_{44} & G_{45} & G_{46} \\ G_{51} & G_{52} & G_{53} & G_{54} & G_{55} & G_{56} \\ G_{61} & G_{62} & G_{63} & G_{64} & G_{65} & G_{66}\end{array}\right)\left(\begin{array}{l}u_{x}^{(0)}+\tilde{F}_{1} \\ u_{y}^{(0)}+\tilde{F}_{2} \\ u_{z}^{(0)}+\tilde{F}_{3} \\ F_{4} \\ \tilde{F}_{5} \\ \tilde{F}_{6}\end{array}\right)$ 


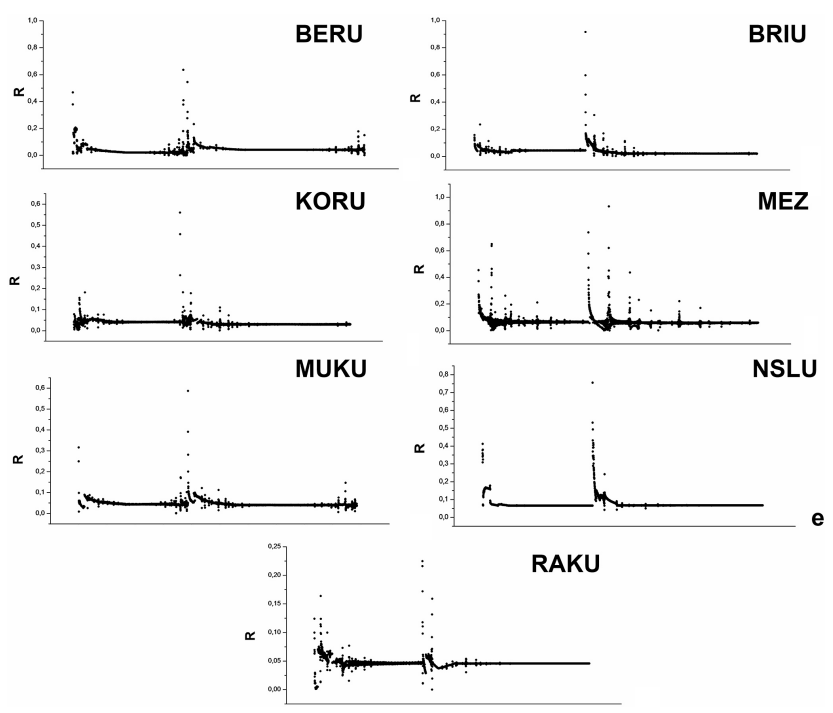

Figure 7. The correlation coefficients for the event which took place near NNP “Synevyr” on 6 January 2012.
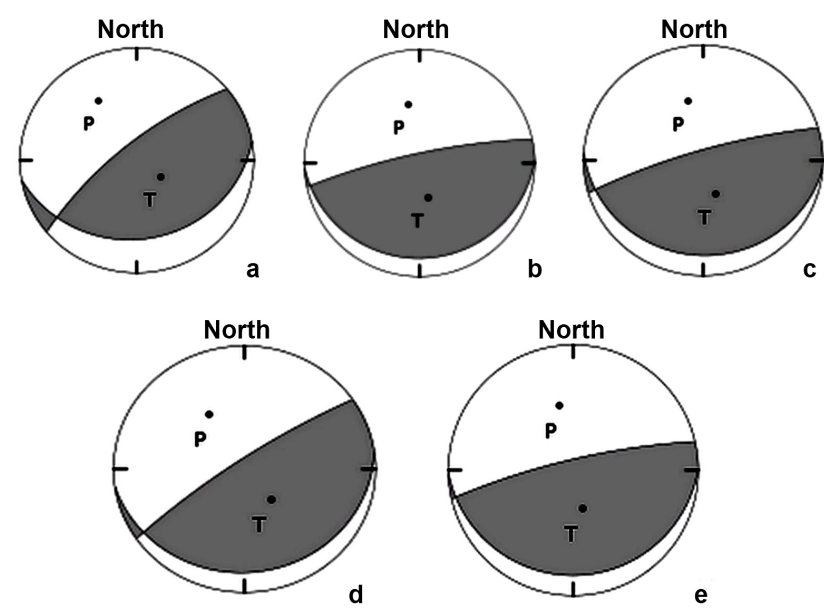

Figure 8. The focal mechanisms of event 6 January 2012 determined by the trial and error method using the real seismograms from seismic stations: (a) BERU; (b) BRIU, MUKU, NSLU, RAKU, (c) MEZ; (d) KORU; (e) average variant of the focal mechanism.

Using only the homogeneous equations is sufficient to get the displacement field on a free surface:

$$
\left\{\begin{array}{l}
G_{11} U_{x}^{(0)}+G_{12} U_{y}^{(0)}+G_{13} U_{z}^{(0)}=-\sum_{i=1}^{6} G_{1 i} \tilde{F}_{i} \\
G_{21} U_{x}^{(0)}+G_{22} U_{y}^{(0)}+G_{23} U_{z}^{(0)}=-\sum_{i=1}^{6} G_{2 i} \tilde{F}_{i} \\
G_{31} U_{x}^{(0)}+G_{32} U_{y}^{(0)}+G_{33} U_{z}^{(0)}=-\sum_{i=1}^{6} G_{3 i} \tilde{F}_{i} .
\end{array}\right.
$$

The stress-displacement discontinuity is determined via the seismic in matrix form of Fryer and Frazer (1984):

$$
F=\left(\begin{array}{l}
-c_{55}^{-1} M_{x z} \\
-c_{44}^{-1} M_{y z} \\
-c_{33}^{-1} M_{z z} \\
p_{x}\left(M_{x x}-c_{13} c_{33}^{-1} M_{z z}\right)+p_{y} M_{x y} \\
p_{x} M_{y x}+p_{y}\left(M_{y y}-c_{23} c_{33}^{-1} M_{z z}\right) \\
p_{x}\left(M_{z x}-M_{x z}\right)+p_{y}\left(M_{z y}-M_{y z}\right)
\end{array}\right) \delta\left(z-z_{s}\right),
$$

where $M_{x x}, M_{y y}, M_{z z}, M_{x z}, M_{y z}, M_{y x}, M_{x y}, M_{z y}$, and $M_{z x}$ are the components of the seismic moment tensor, and $c_{13}$, $c_{23}, c_{33}, c_{44}$, and $c_{55}$ are the components of the stiffness matrix.

As a result, the displacement field of the free surface of an anisotropic medium is in the spectral domain as

$U=\left(U_{x}^{0}, U_{y}^{0}, U_{z}^{0}\right)^{T}=\left(\mathbf{G}^{13}\right)^{-1} \cdot y$,

where $\mathbf{G}^{13}=\left(\begin{array}{lll}G_{11} & G_{12} & G_{13} \\ G_{21} & G_{22} & G_{23} \\ G_{31} & G_{32} & G_{33}\end{array}\right)$,

$y=-\left(\sum_{i=1}^{6} G_{1 i} \tilde{F}_{i}, \sum_{i=1}^{6} G_{21 i} \tilde{F}_{i}, \sum_{i=1}^{6} G_{3 i} \tilde{F}_{i}\right)^{T}$,

$a=-\left(G_{11} \tilde{F}_{1}+G_{12} \tilde{F}_{2}+G_{13} \tilde{F}_{3}+G_{14} \tilde{F}_{4}+G_{15} \tilde{F}_{5}+G_{16} \tilde{F}_{6}\right)$,

$b=-\left(G_{21} \tilde{F}_{1}+G_{22} \tilde{F}_{2}+G_{23} \tilde{F}_{3}+G_{24} \tilde{F}_{4}+G_{25} \tilde{F}_{5}+G_{26} \tilde{F}_{6}\right)$,

$c=-\left(G_{31} \tilde{F}_{1}+G_{32} \tilde{F}_{2}+G_{33} \tilde{F}_{3}+G_{34} \tilde{F}_{4}+G_{35} \tilde{F}_{5}+G_{36} \tilde{F}_{6}\right)$.

Using Eq. (11) and three-dimensional Fourier transform, we obtain a direct problem solution for the displacement field of the free surface of an anisotropic medium in the time domain as

$$
\begin{array}{r}
\boldsymbol{u}\left(x, y, z_{R}, t\right)=\frac{1}{8 \pi^{3}} \iiint_{-\infty} \omega^{2} U\left(p_{x}, p_{y}, z_{R}, \omega\right) \\
e^{j \omega\left(t-p_{x} x-p_{y} y\right)} \mathrm{d} p_{x} \mathrm{~d} p_{y} \mathrm{~d} \omega,
\end{array}
$$

where $z_{R}$ is the epicentral distance, and $p_{x}, p_{y}$ are the horizontal slowness.

\section{Inverse problem}

It is known that inverse problems are inherently incorrect. In seismology methods and approaches often are used, which are reduced to the selection of the physical characteristics of the studied environment or earthquake (Brace, 1978; Clinton et al., 2006; Cohn et al., 1982; Hartzell and Heaton, 1979; Santosa and Symes, 1986; Hanks and and Kanamori, 1979; Honda, 1957, 1962; Scholz et al., 1973). The development of 


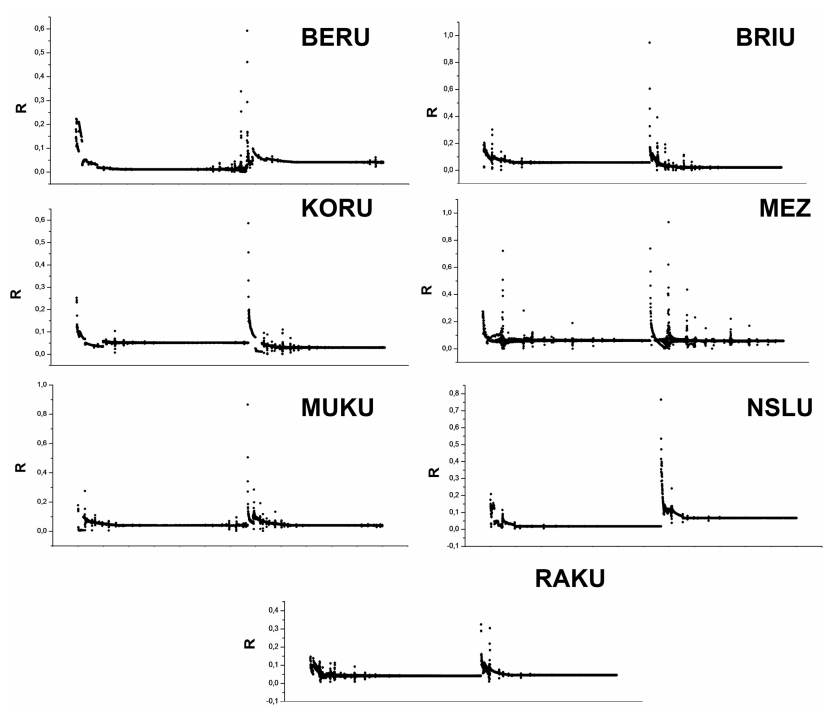

Figure 9. Pearson's correlation coefficients between synthetic and real seismograms for the event which took place near NNP "Synevyr" on 10 January 2012.

new methods and algorithms for determining source parameters is a relevant and important issue. Of course, there is no general and reliable approach.

Furthermore, it is impossible to consider all effects in modelling wave processes during propagation of seismic waves in heterogeneous environments. Therefore, for an anisotropic medium it is difficult to construct a theory that would be based only on analytical expressions. Thus, we must resort to numerical solution of equations.

\subsection{Traditional graphic method and trial and error method for determining the earthquake source parameters}

The focal mechanism solution for earthquakes in regions of low seismic activity today is the actual problem. Particularly it is very important for the Carpathian region of Ukraine, where there is an insufficient number of stations in addition to low seismic activity. It is impossible to determine a focal mechanism with software packages.

The software package Matlab 7.12.0 (R2011a) is used for programming in this paper. Algorithms and software of the direct dynamic problem solving are based on the method described in Sect. 2.1. This method is based on the use of matrix and wave propagators, which are applied to inhomogeneous anisotropic media modelled by a bundle of homogeneous anisotropic layers with parallel boundaries. The algorithm for calculating the displacement field on the free surface of a layered anisotropic medium in the Cartesian coordinate system $\left(u_{x}^{(0)}, u_{y}^{(0)}, u_{z}^{(0)}\right)$ is based on certain physical and mathematical constraints:
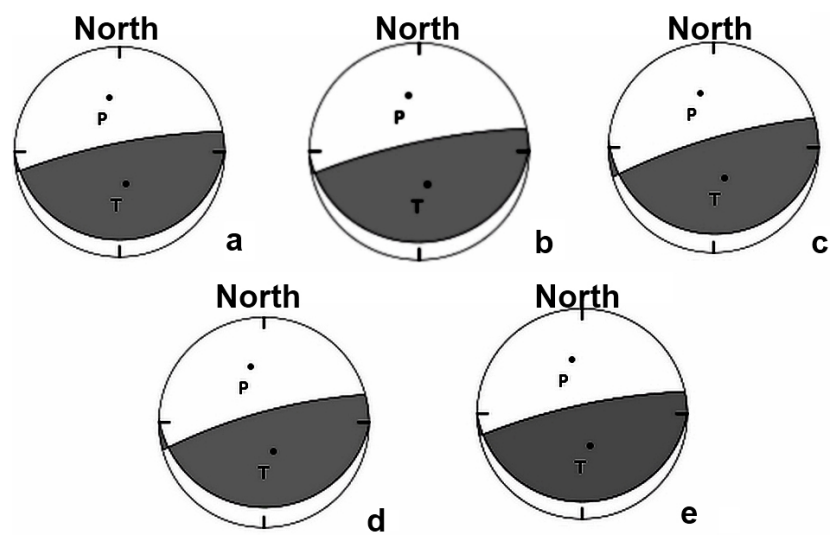

Figure 10. The focal mechanisms of event 10 January 2012 determined by the trial and error method using the real seismograms from seismic stations: (a) BERU; (b) BRIU, MUKU, NSLU, KORU, (c) MEZ; (d) RAKU; (e) average variant of the focal mechanism.

1. Heterogeneous anisotropic medium is modelled by a bundle of homogeneous anisotropic layers.

2. Homogeneous anisotropic layers are separated by parallel boundaries.

3. Contact between the layers is considered hard (continuity of displacements and stresses).

4. A point source is located within any homogeneous anisotropic layer.

Thus, the algorithm for calculating the displacement field on the free surface of a layered anisotropic medium is defined by Eq. (12) in the Cartesian coordinate system. The equations for the direct dynamic problem are obtained by means of numerical calculations.

In the algorithms and programs fast Fourier transform to the variables $(t, \omega)$ is used. Maximum frequency, step frequency and sampling time were chosen from the following conditions:

$\omega_{\max }=2 \pi f_{\max }$,

where $\Delta t=\frac{1}{2 f_{\max }} ; \Delta f=\frac{f_{\max }}{2^{n}}(n=10)$.

Waves are excited by a point source, represented by a seismic moment tensor. The relationship between the components of the seismic moment tensor and fault plane orientation angles is given as in Aki and Richards (2002):

$$
\begin{gathered}
M_{x x}=-M_{0}\left(\sin \delta \cos \lambda \sin 2 \varphi_{s}+\sin 2 \delta \sin \lambda \sin ^{2} \varphi_{s}\right) \\
M_{x y}=M_{0}\left(\sin \delta \cos \lambda \cos 2 \varphi_{s}+1 / 2 \sin 2 \delta \sin \lambda \sin 2 \varphi_{s}\right) \\
M_{x z}=-M_{0}\left(\cos \delta \cos \lambda \cos \varphi_{s}+\cos 2 \delta \sin \lambda \sin \varphi_{s}\right)=M_{z x} \\
M_{y y}=M_{0}\left(\sin \delta \cos \lambda \sin 2 \varphi_{s}-\sin 2 \delta \sin \lambda \cos ^{2} \varphi_{s}\right) \\
M_{y z}=-M_{0}\left(\cos \delta \cos \lambda \sin \varphi_{s}-\cos 2 \delta \sin \lambda \cos \varphi_{s}\right)=M_{z y} \\
M_{z z}=-M_{0} \sin 2 \delta \sin \lambda
\end{gathered}
$$

where $M_{0}=\mu A u(\tau)$ is the seismic moment; $\delta$ is the dip angle; $\varphi_{\mathrm{s}}$ is the strike angle; $\lambda$ is the slip angle. 


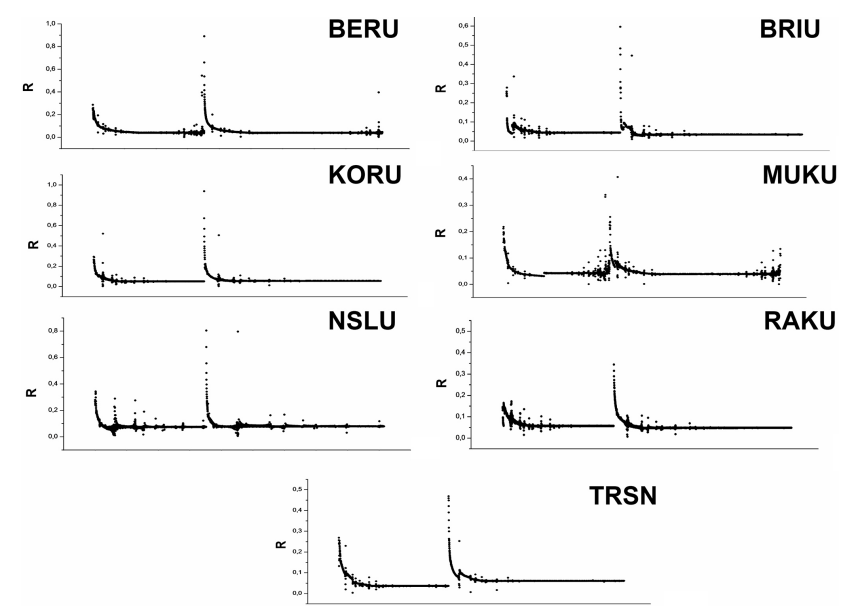

Figure 11. Pearson's correlation coefficients between synthetic and real seismograms for the event which took place near village Ugla on 24 October 2012.
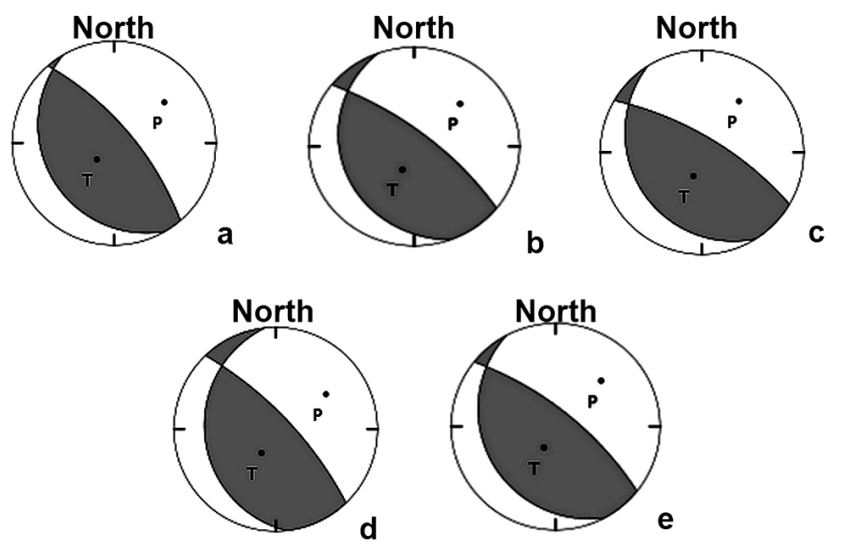

Figure 12. The focal mechanisms of event 24 October 2012 determined by the trial and error method using the real seismograms from seismic stations: (a) BERU, BRIU, KORU, TRSN; (b) NSLU, (c) MUKU; (d) RAKU; (e) average variant of the focal mechanism.

The tensor (Eq. 13) is defined by the geometric orientation of the fault plane and the value of seismic moment $M_{0}$.

Obtaining the analytical expressions to determine the earthquake source parameters, when the source is represented by seismic moment tensor, is difficult. The most accurate results of the inverse problem solving for the source parameters are obtained by the trial and error method. In this method, the synthetic seismograms are calculated many times for all possible combinations of orientation angles of the fault plane and the velocity model for the Carpathian region of Ukraine. The correlation coefficients are calculated for all these synthetic seismograms and real record of events. The biggest correlation coefficient corresponds to the most probable combination of orientation angles of the fault plane. The best results of solving are for the records from stations in smaller epicentre distance; these records usually have the

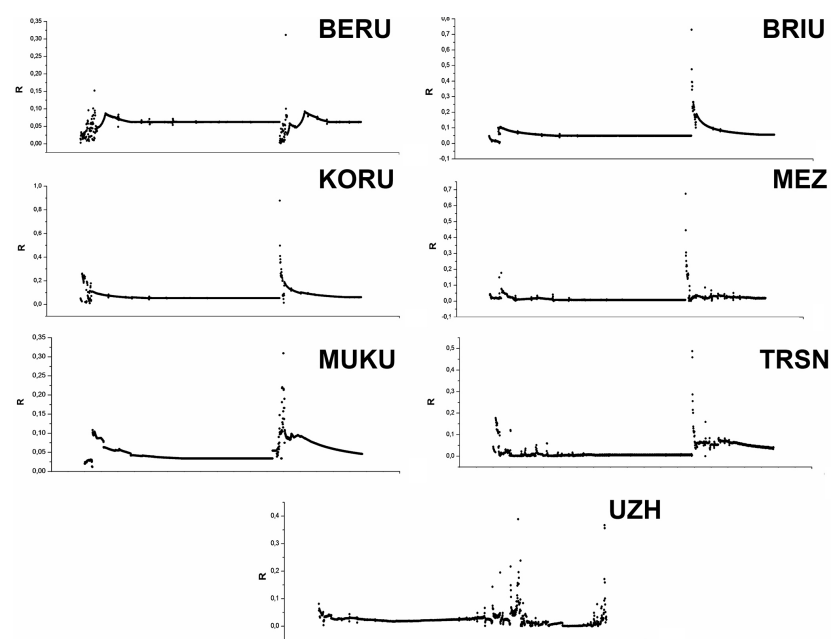

Figure 13. Pearson's correlation coefficients between synthetic and real seismograms for the event which took place near village Nyzhnje Selyshche on 4 April 2013.
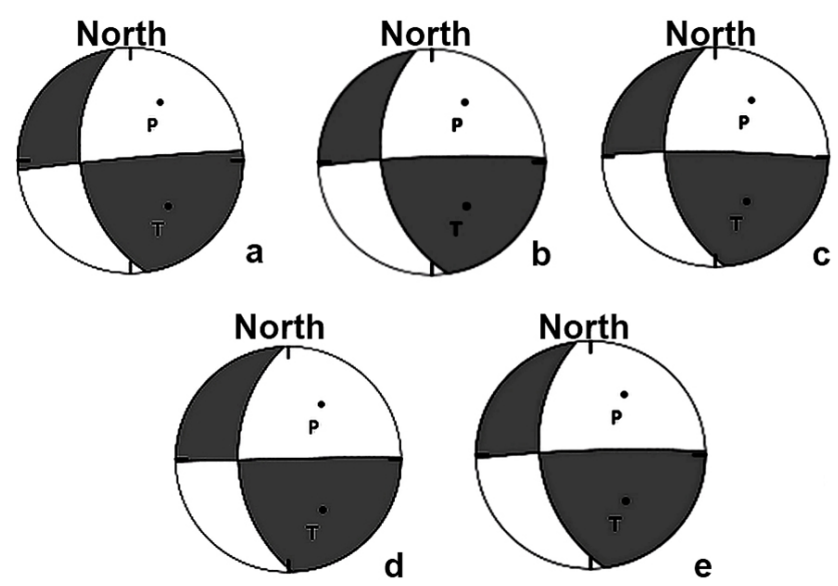

Figure 14. The focal mechanisms of event 4 April 2013 determined by the trial and error method using the real seismograms from seismic stations: (a) BERU; (b) BRIU, KORU, MEZ, TRSN; (c) MUKU; (d) UZH; (e) average variant of the focal mechanism.

lowest noise level. The results obtained by this method are compared with results for the same event but received by graphical method (Malytskyy et al., 2013).

In the trial and error method the matrix in Eq. (11) is solved for the velocity model for the Carpathian region of Ukraine (Table 1) and for the stress-displacement discontinuity (Eq. 10), where components of seismic tensor are determined via oriental angles of the fault plane (Eq. 13).

The traditional graphical method is based on the first arrival P-waves (Malytskyy et al., 2013; Baumbach and Bormann, 2011) using information about fuzzy first motion (Cronin, 2004) and the S / P amplitude ratio (Hardebeck and Shearer, 2003). 
The polarities of first motion P-waves were defined from complete records of seismograms taking into account the possible inversion of the sign on the $z$ component. A logarithm of the amplitude ratio $\mathrm{S} / \mathrm{P}$ is calculated using data from the three components of seismic records of this event at each station (Hardebeck and Sharer, 2003; de Natale, 1994). Input data for the azimuth and take-off angle are calculated with software packages for each event.

Most often an approach is used where nodal planes are plotted on a lower-hemisphere stereographic projection such as to best fit the polarities of first arrivals of P-waves at the location of a station polarity on the projection depending on the station azimuth and take-off angle of the ray of first arrival connecting the source and the station.

These focal mechanisms are determined using a method that attempts to find the best fit to the direction of P-wave first motions observed at each station. For a double-couple source mechanism (or only shear motion on the fault plane), the compression first motions should lie only in the quadrant containing the tension axis, and the dilatation first motions should lie only in the quadrant containing the pressure axis. Accuracy of focal mechanism solution depends on the input data: velocity model and coordinate of the hypocentre (they determine the take-off angle), quality of seismic records and sign inversion on the seismometer, so that "up" is "down" (they determine character entry wave).

$\mathrm{S} / \mathrm{P}$ amplitude ratios are applicable because of $\mathrm{P}$-wave amplitude being the largest on $P$ and $T$ axes of focal mechanism and the smallest near the nodal planes, while the $S$-wave amplitude is the largest near the nodal planes. S / P amplitude ratios with a wide range of values can more accurately constrain the location of seismic station projections on the focal sphere. The larger the S / P amplitude ratios, the closer the location of the seismic station projection to the nodal line.

Seismic moment and other spectral parameters are computed by Eqs. (12)-(19) for each station (Baumbach and Bormann, 2011).

The seismic moment is computed according to

$M_{0}=4 \pi r v_{p}^{3} \rho u_{0} /\left(\theta S_{a}\right)$,

where $r$ is the hypocentral distance, $v_{p}$ is the P-wave velocity, $\rho$ is the density, $u_{0}$ is the low-frequency level (plateau) of the displacement spectrum, $\Theta$ is the average radiation pattern and $S_{\mathrm{a}}$ is the surface amplification for P-waves.

The source radius $R$ is computed from the relationship

$R=\frac{3.36 v_{p}}{2 \sqrt{3} \pi f_{\mathrm{c}}}$,

where $f_{\mathrm{c}}$ is corner frequency of the $\mathrm{P}$-wave.

The size of the circular rupture plane is computed as

$A=\pi R^{2}$.

The average source dislocation is according to

$\bar{D}=M_{0} / \mu A$, where the shear modulus is computed by

$\mu=v_{p}^{2} \rho / 3$.

The stress drop, seismic energy and magnitude ML are computed according to

$\Delta \sigma=7 M_{0} / 16 R^{3}$,
$E_{\mathrm{s}}=M_{0} \cdot 1.6 \times 10^{-5}$,
$M L=\left(\lg E_{\mathrm{s}}-4\right) / 1.8$.

\subsection{Determining the parameters of earthquake sources}

In approving the proposed trial and error method for determining the earthquake source parameters the four events in the Carpathian region of Ukraine were considered. For each of these events an earthquake focal mechanism is determined by the trial and error method and graphic method. These focal mechanisms are compared.

In this paper seismic events are considered:

1. The earthquake took place in the district National Nature Park (NNP) "Synevyr" in the Carpathian region of Ukraine $\left(\varphi=48.5309^{\circ}, \lambda=23.8365^{\circ}, \mathrm{ML}=2.53\right)$, 6 January 2012 at 04:34:10.464 UTC at the depth $5 \mathrm{~km}$.

2. The earthquake took place in the district NNP "Synevyr" in the Carpathian region of Ukraine $\left(\varphi=48.5367^{\circ}, \lambda=23.8378^{\circ}, \mathrm{ML}=2.68\right), 2012.01 .10$ at $12: 12: 55584$ at the depth $5.7 \mathrm{~km}$.

3. The earthquake took place near village Ugla $\left(\varphi=48.1676^{\circ}, \lambda=23.6525^{\circ}, \mathrm{ML}=2.43\right), 2012.10 .24$ at 03:13:40501 at the depth $5 \mathrm{~km}$.

4. The earthquake took place near village Nyzhnje Selyshche $\left(\varphi=48.1977^{\circ}, \lambda=23.4663^{\circ}, \mathrm{ML}=2.56\right)$, 2013.04.04 at 21:15:1436 at the depth $1.8 \mathrm{~km}$.

Location map of the projection of seismic stations in the Carpathian region of Ukraine and specified epicentre of the four events in the Carpathian region of Ukraine is shown in Fig. 2. Spectral parameters for the events in the Carpathian region of Ukraine calculated by Eqs. (12)-(19) are shown in Table 2.

The first step of solving the inverse problem of seismology is determining the parameters of earthquake source by graphic method. The data for the determining the focal mechanism by the traditional graphic method are a sign of first arrival of P-wave, azimuth from epicentre on the seismic station, take-off angle and the $\mathrm{S} / \mathrm{P}$ amplitude ratio on each seismic record of this event. The input data for graphic method are given in Tables 3-6 for all the seismic events.

The second step of solving the inverse problem of seismology is determining the parameters of earthquake source by the trial and error method. Using the velocity model for the Carpathian region of Ukraine (Table 1), the wave field on 
a free surface (Eq. 12) is calculated many times for all combinations of dip $(\delta)$, strike $\left(\varphi_{\mathrm{s}}\right)$ and slip $(\lambda)$ angles. All of these synthetic seismograms are compared with real records of this event. Pearson's correlation coefficients are calculated for all of these synthetic waveforms and real seismograms on each seismic station which record this event. The largest coefficient corresponds to the most probable combination of fault plane orientation angles $\left(\varphi_{\mathrm{s}}, \delta, \lambda\right)$. In Fig. 7 Pearson's correlation coefficients between synthetic waveforms and real seismograms on seismic stations (BERU, BRIU, KORU, MUKU, MEZ, NSLU, and RAKU) are shown. On the horizontal axis a nodal plane identifier (combinations of dip, strike and slip angles) is plotted. The maximum coefficient $R$ corresponds to the most probable focal mechanism. In Fig. 8. the focal mechanisms determined by the trial and error method using real seismograms from different seismic stations and average variant of the focal mechanism of event 6 January 2012 are shown.

The focal mechanism of event 10 January 2012 is also determined by the above-described trial and error method. Similarly to the previous case the synthetic seismograms and correlation coefficients are calculated. In Fig. 9 the coefficients $R$ between synthetic and real seismograms are shown. On the horizontal axis a nodal plane identifier (combinations of dip, strike and slip angles) is plotted. The maximum correlation coefficient $R$ corresponds to the focal mechanism. In Fig. 10. the focal mechanisms determined by the trial and error method using real seismograms from different seismic stations and average variant of the focal mechanism of event 10 January 2012 are shown.

The focal mechanism of event 24 October 2012 is also determined by the trial and error method. Similarly to the previous cases the synthetic seismograms and correlation coefficients are calculated. In Fig. 11 Pearson's correlation coefficients $R$ between synthetic and real seismograms are shown. On the horizontal axis a nodal plane identifier (combinations of dip, strike and slip angles) is plotted. The maximum correlation coefficient $R$ corresponds to the focal mechanism. In Fig. 12 the focal mechanisms determined by the trial and error method using real seismograms from different seismic stations and average variant of the focal mechanism of event 24 October 2012 are shown.

The focal mechanism of event 4 April 2013 is determined by the trial and error method. In Fig. 13 the coefficients $R$ for synthetic and real seismograms are shown. On the horizontal axis a nodal plane identifier (combinations of dip, strike and slip angles) is plotted. In Fig. 14 the focal mechanisms determined by the trial and error method using real seismograms from different seismic stations and average variant of the focal mechanism of event 4 April 2013 are shown.

The third step is comparative analysis between the focal mechanisms obtained by different methods. Comparing the focal mechanisms determined by graphic method and trial and error method of the event 6 January 2012 (Figs. 3b, 8e), the event 10 January 2012 (Figs. 4b, 10e); the event
24 January 2012 (Figs. 5b, 12e) and the event 4 April 2013 (Figs. 6b, 14e), we can conclude that the results obtained by different methods are similar. Thereby, these two methods can be used for determining the focal mechanisms of the local events.

\section{Conclusions}

The results of this paper contribute to the fundamental understanding of wave propagation in anisotropic media. A numerical technique for computing synthetic seismograms has been developed in the framework of the theory. Wave propagation in multilayer media requires that displacement and stress vectors be continuous everywhere, including the interfaces.

Seismologists have been able to invert the rupture process of a number of earthquakes, and many of the features predicted by simple dynamic source models have been quantified and observed. Foremost among these is the shape of the FF spectrum, the basic scaling laws relating particle velocity and acceleration to properties of the fault, such as size, stress drop and rupture velocity. Recent inversions of earthquake slip distributions using kinematic source models have found very complex source distributions that require an extensive reappraisal of classical source models. It is shown that the developed method for determining the earthquake parameters can be used successfully using real records. It should be also noted that the proposed method for determining the seismic moment tensor can be used in seismology for a class of problems, when the velocity model of the medium is known. Thus, the methods, approaches, algorithms, software for the propagation of seismic waves and results of direct and inverse dynamic problems of seismology proposed and developed by the authors and highlighted in the paper can be successfully used in the study of the seismic regions and effective implementation in the construction of the earthquake source mechanism which is crucial for seismic regions of the Ukraine.

The focal mechanisms are determined also using the graphical method, which is based on the first arrival Pwaves, information about fuzzy first motion and the S / P amplitude ratio.

The advantage of the trial and error method is the possibility of using it for determining the focal mechanism in the case of a small number of seismic stations which record this event.

Edited by: L. Eppelbaum 


\section{References}

Aki, K. and Richards, P. G.: Quantitative Seismology, 2nd Edn., University Science Books, 2002.

Babuska, V.: Anisotropy of $\mathrm{Vp}$ and $\mathrm{Vs}$ in rock-forming minerals, Geophys. J. R. Astr. Soc., 50, 1-6, 1981.

Bachman, R. T.: Acoustic anisotropy in marine sediments and sedimentary rocks, J. Geophys. Res., 84, 7661-7663, 1979.

Backus, G. E.: Long-wave elastic anisotropy produced by horizontal layering, J. Geophys. Res., 67, 4427-4440, 1962.

Baumbach, M. and Bormann, P.: Determination of source parameters from seismic spectra, Potsdam: Deutsches GeoForschungsZentrum GFZ, 2011.

Behrens, E.: Sound propagation in lamellar composite materials and averaged elastic constants, J. Acoust. Soc. Am., 42, 168-191, 1967.

Brace, W. F.: Volume changes during fracture and frictional sliding, A Rev. Pure Appl. Geoph., 116, 603-614, 1978.

Bullen, K. E.: On strain energy in Earth's upper mantle, Trans. Am. Geophys. Union., 34, 107-116, 1953.

Cerveny, V.: Seismic ray theory, Cambridge University Press, 2001.

Chapman, C. H.: Fundamentals of seismic wave propagation, Cambridge University Press, 608, 2004.

Clinton, J. F., Hauksson, E., and Solanki, K.: An evaluation of the scan moment tensor solution: robustness of the MW Magnitude scale, style of faulting and automation of the method, Bull. Seismol. Soc. Am., 96, 1689-1705, 2006.

Cohn, S. N., Hong, T., and Helmberger, D. V.: The Oroville earthquakes: a study of source characteristics and site effects, J. Geophys. Res., 87, 4585-4594, 1982.

Cronin, V. A.: A draft primer on focal mechanism solutions for geologists: Teaching Quantitative Skills in the Geosciences, available at: http://serc.carleton.edu/files/NAGTWorkshops/ structure04/Focal_mechanism_primer.pdf, 2004.

Dunkin, I. W.: Computation of modal solutions in layered elastic media at high frequencies, Bull. Seismol. Soc. Am., 55, 335358,1965

Fryer, G. J. and Frazer, L. N.: Seismic waves in stratified anisotropic media, Geophys. J. R. Astr. Soc., 78, 691-710, 1984.

Fryer, G. J. and Frazer L. N.: Seismic waves in stratified anisotropic media.-II. Elastodynamic eigensolutions for some anisotropic systems, Geophys. J. R. Soc., 91, 73-101, 1987.

Fuchs, K.: Explosion seismology and the continental crust-mantle boundary, J. Geol. Soc., 134, 139-151, 1977.

Hanks, T. C. and Kanamori, H.: A moment-magnitude scale, J. Geophys. Res., 84, 2348-2350, 1979.

Hardebeck, J. L. and Shearer, P. M.: Using S/P Amplitude Ratios to Constrain the Focal Mechanisms of Small Earthquakes, Bull. Seismol. Soc. Am., 93, 2434-2444, 2003.

Hartzell, S. H. and Heaton, T. H.: Inversion of strong ground motion and teleseismic waveform data for the fault rupture history of the 1979 Imperial Valley. California earthquake, Bull. Seismol. Soc. Am., 73, 1553-1583, 1983.
Hartzell, S. H. and Heaton, T. H.: Inversion of strong ground motion and teleseismic waveform data for the fault rupture history of the 1979 Imperial Valley. California earthquake, Bull. Seismol. Soc. Am., 73, 1553-1583, 1983.

Haskell, N. A.: The dispersion of waves in multilayered media, Bull. Seism. Soc. Am., 43, 17-34, 1953.

Honda, H.: The mechanism of the earthquakes, Sci. Rep. T6hoku Univ., Geophysics, 9 (Suppl.), 1-46, 1957.

Honda, H.: Earthquake mechanism and seismic waves, J. Phys. Earth, 10, 1-97, 1962.

Ilan, A., Ungar, A., and Alterman, Z. S.: An improved representation of boundary conditions in finite difference schemes for seismological problems, Geophys. J. Roy. Astron. Soc., 43, 727-745, 1975.

Kennett, B. L. N.: Seismic waves in laterally inhomogeneous media, Geophys. J. R. Astr. Soc., 27, 301-325, 1972.

Kennett, B. L. N.; Seismic wave propagation in stratified media, Cambridge Univ. Pres., Cambridge, 1983.

Malytskyy, D.: General principles of seismological dynamic problem solution on the basis of the recurrent method, Geophys. J., IGF NASU, 20, 96-98, 1998.

Malytskyy, D.: Analytic- Numerical Approaches to the Calculation of Seismic Moment Tensor as a Function of Time, Geoinformatika, 1, 79-85, 2010.

Malytskyy, D., Hrytsai, O., and Muyla, O.: The features of the construction of the focal mechanisms of local earthquakes using the example of an event near Beregovo 23.11.2006, Visnyk KNU, Geology, 60, 37-42, 2013.

Malytskyy, D. and Muyla, O.: Particularities of modelling of earthquake source, Theor. Appl. Aspects Geoinform., 1, 304-310, 2008.

de Natale, G.: Focal mechanism determination for volcanic microearthquakes, Annali di geofisica, XXXVII, 1621-1643, 1994.

Santosa, F. and Symes, W. W.: Inverse problem for a layered acoustic wave propagation. Mathematical and Computational Methods in Seismic Exploration and Reservoir Modeling, edited by: Fitzgibbon, W., SIAM Publications, Philadelphia, 259-263, 1986.

Scholz, C. H., Sykes, L. R., Aggarawal, Y. P.: Earthquake prediction: A physical basis, Science. 181, 803-810, 1973.

Thomsen, L.: Weak elastic anisotropy, Geophysics, 51, 1954-1966, 1986.

Thomson, W. T.: Transmission of elastic waves through a stratified solid medium, J. Appl. Phys., 21, 89-93, 1950.

Ursin, B.: Review of elastic and electromagnetic wave propagation in horizontally layered media, Geophysics, 48, 1063-1081, 1983.

Woodhouse, J. H.: Asymptotic results for elastodynamic propagator matrices in plane stratified and spherically stratified Earth models, Geophys. J. R. Astr. Soc., 54, 263-280, 1978.

Yang, D. H., Liu, E., Zhang, Z. J., and Teng, J.: Finite-difference modelling in two-dimensional anisotropic media using a fluxcorrected transport technique, Geophys. J. Int., 148, 320-328, 2002.

Zahradnik, J.: Finite difference solutions to certain diffraction problems, Studia Geophys. Geod., 19, 233-244, 1975. 